\title{
Hepatitis a and Japanese encephalitis targeting specific adult age group preventable by vaccination
}

\begin{abstract}
Spring, with its warm weather in Republic of Korea, is a good season for outdoor activities such as picnics and travels but is also a good season for infections to spread. Having people keep sanitary guidelines guides such as washing hands and coughing with their mouth closed an arm around is important in prevention of the spread, but the safest way is to get people vaccinated. There are about 20 diseases that can be prevented by vaccination. Among them there are diseases that occur more frequently in certain age groups. Hepatitis A and Japanese Encephalitis are two big examples.
\end{abstract}

Volume I Issue 4 - 2017

\author{
Byungwook Yoo, David Samuel Kwak \\ Department of Family Medicine, Soonchunhyang University \\ College of Medicine, Korea
}

\begin{abstract}
Correspondence: Byungwook Yoo, Department of Family Medicine, Soonchunhyang University Seoul Hospital, Soonchunhyang University College of Medicine, Korea, Tel +822-709-9158, Fax +82-2-709-9133,

Email byungwookyoo@hanmail.net
\end{abstract}

Received: August 25, 2017| Published: December 06, 2017

\section{In the past 5 years, $70 \%$ of hepatitis a patients were young adults in their 20's and 30's}

\section{Prevention is important because there is no curative medicine}

Hepatitis A infections occur worldwide and generate approximately 1.4 million patients annually. Large or sporadic spreads are usually caused by inadequate cooking or drinking water from tainted sources. An epidemic occurred in Shanghai in 1988 that resulted in infection of 300,000 patients. Clam was found to be the source of the infection. ${ }^{1-}$ ${ }^{3}$ The spread of hepatitis A virus is closely related to the economic level and public hygiene of a population. In the past, when economic level of Korea was low, people were more easily exposed to situations that led to infections of the virus. In that time, they often went through adolescence obliviously infected of the disease and naturally attained antibodies. ${ }^{1}$ Due to improvements in public health and living conditions as the economy grew, however exposure to the virus decreased significantly, reducing natural immunity in young people. ${ }^{1}$ In fact, in analyses of 10,696 hepatitis A patients from the past 5 years, $7 \%$ were in their teens, $29 \%$ in 20 's, $43 \%$ in 30 's, and $16 \%$ in their 40 's. ${ }^{2}$ Adult patients in their 20's and 30's who were not vaccinated during their infancy were more commonly found than in other age groups. Hepatitis A usually passes without a symptom when infected under before 6 years of age, but when it is infected during adulthood, up to $70 \%$ of patients show jaundice and a small number may also have acute renal failure, cholecystitis and pancreatitis. In rare cases atypical symptoms such as recurrent infections and autoimmune infections may occur. ${ }^{3}$ Since hepatitis A does not have any cure, prevention is the most important. Adults may need to be vaccinated if they have been properly hygienic but have never either been vaccinated against or infected of hepatitis A. 2 injections are needed for the vaccination, 6 months or more apart (Table 1$){ }^{4}$

Table I Number of type hepatitis A infection occurrences in the past 5 years (20I I-2015). ${ }^{2}$

\begin{tabular}{llllllll}
\hline \multicolumn{6}{l}{ Number of hepatitis a infection occurrences in the past $\mathbf{5}$ years (unit: persons) } \\
\hline Under 10 & $19-$-Oct & $20-29$ & $30-39$ & $40-49$ & $50-59$ & $60-69$ & Above 70 \\
84 & 683 & 3,092 & 4,593 & 1,761 & 296 & 87 & 100 \\
\hline
\end{tabular}

Japanese encephalitis; incidence and death rate high among people from 40 to 70 .

\section{Adults can be vaccinated with I injection}

Japanese encephalitis is an infection showing acute neurologic symptoms after a patient is bitten by a mosquito called Culex tritaeniorhynchus containing the virus. When infected, most people go through the disease without a specific symptom, but 1 in 250 people show clinical symptoms. ${ }^{5,6}$ Many of those people would also have neurologic sequel even after recovery, and as many as 3 in 10 people would result in death. ${ }^{7}$ Number of Japanese encephalitis patients have increased 13 folds in the past 5 years making it the fastest growing infective disease spread by mosquito in Korea. ${ }^{8}$ This is thought to be due to the increased number of travelers to South East Asia during summer season, in addition to the changes in ecosystem such as in

global warming providing a safe atmosphere for mosquito population to stay amass. Japanese encephalitis has highest incident rate among people aged 40 and up. According to the statistical data of incident population according to year and age acquired by Korea Health Insurance Review \& Assessment Service, in the past 5 years, people above 40 comprised $87 \%$ of the total number of Japanese encephalitis patients (Table 2). ${ }^{9}$

The problem is that the average age for patients resulting in death from Japanese encephalitis is 52.1, meaning that adults are in greater danger for the worst result. This can be thought to be due to the lack of antibodies in people aged above 40, born before 1971 that were probably not vaccinated against Japanese encephalitis because it is the year Japanese encephalitis vaccination was first introduced to Korea. In addition, the decreasing antibody positivity rate in people above 40 is thought to affect the result as well. A study of Korean adults showed 
that antibody positivity rate in people ages from 15 to 29 was $95 \%$ and the rate started to decrease in ages 30 and up, down to $75.24 \%$ in the age group 55 to 59 , and $59.77 \%$ in people 70 years or above. ${ }^{10}$ Fortunately, Japanese encephalitis is also preventable by vaccination. Last year, Ministry of Food and Drug Safety, for the first time in Korea, gave permission to live vaccination of Japanese encephalitis inject able on adults. It is more convenient that it only needs 1 injection for prevention, compared to older inactivated vaccines which needed 3 injections. Korea Centers for Disease Control and Prevention recommends adults that have plans to visit epidemic countries, who have not received vaccinations for Japanese encephalitis, to be vaccinated. WHO (World Health Organization) also recommends vaccination to those in need that have plans to visit countries of high rate of occurrence during seasons Japanese encephalitis is easily transmitted. Even though the disease is preventable, danger of mass infection in vulnerable age group exists due to low awareness of vaccination. The best preventive method against infective disease is to complete necessary vaccinations in the appropriate time accordingly to the age. Because Japanese encephalitis, growing in numbers recently, is also showing potential to spread even more rapidly in vulnerable age group like hepatitis A is, prevention of the diseases by vaccination is important.

Table 2 Number of incidents in middle-aged people 40 and above in the past 5 years (20II-20I5). ${ }^{9}$

\begin{tabular}{lccccc}
\hline Japanese encephalitis patients ages & $\mathbf{4 0 - 7 0}$ in the past $\mathbf{5}$ years (unit : persons) \\
\hline & $\mathbf{2 0 I I}$ & $\mathbf{2 0 I 2}$ & $\mathbf{2 0 1 3}$ & $\mathbf{2 0 1 4}$ & $\mathbf{2 0 1 5}$ \\
\hline Total number of patients & 3 & 20 & 14 & 26 & 40 \\
Patients aged 40-70 & 2 & 17 & 14 & 23 & 37 \\
Percentage of patients aged 40-70 & $67 \%$ & $85 \%$ & 100 & 88 & $93 \%$ \\
\hline
\end{tabular}

\section{Acknowledgements}

None.

\section{Conflict of interest}

The author declares no conflict of interest.

\section{References}

1. Hwa Young Lee. Incidence and Epidemiology of Japanese Encephalitis in Korea, Public Health Weekly Report, KCDC. 2010;8(19):1-11.

2. Prevention Infective Disease Web Statistical System-Statistics par diseases; Hepatitis A, years 2011-2016.

3. Dynamics and Management of Immunization Targeted Infective Diseases, Chapter 20 Hepatitis A, Korea.

4. Korea Centers for Disease Control and Prevention. Guideline on preventive measures for Hepatitis B infection during pregnancy for medical facilities. 2014.
5. Korea Centers for Disease Control and Prevention Infective Disease Web Statistical-Statistics par diseases; Hepatitis B, years 2011-2016.

6. Korea Centers for Disease Control and Prevention, Dynamics and Management of Immunization Targeted Infective Diseases, Chapter 5 Hepatitis B.

7. Prevention, Dynamics and Management of Immunization Targeted Infective Diseases, Chapter 14 Japanese Encephalitis.

8. Prevention Infective Disease Web Statistical System-Statistics par diseases; Individual searches: Japanese Encephalitis, Malaria, Yellow Fever, Dengue Fever, and West Nile Fever.

9. Korea Centers for Disease Control and Prevention Infective Disease Web Statistical System-Statistics par diseases; Japanese Encephalitis, years 2011-2016.

10. Yun SW, Lee WK, Cho SY, et al. The Seroprevalence Rate, Vaccination Rate and Seroconversion Rate of Hepatitis A in Central Region of Korea. Korean J Gastroenterol. 2011;57(3):166-172. 